\title{
A New Approach in the Use of Multimedia for Technology Enhanced Learning
}

\author{
Haniph A. Latchman ${ }^{1}$ and Denis Gillet ${ }^{2}$ \\ 'Electrical and Computer Engineering Department University of Florida Gainesville FL 32611 \\ ${ }^{2}$ Swiss Federal Institute of Technology CH 1015 Lausanne Switzerland \\ latchman@list.ufl.edu
}

\begin{abstract}
In this paper we describe ongoing work in the Electrical and Computer Engineering Department at the University of Florida as we offer an Online MS degree in Electrical Engineering and as we proceed with the development of major components of a BS degree in Electrical Engineering. In particular we describe the implementation of the "lectures on demand" method using multimedia streaming technologies within the now widely accepted Asynchronous Learning Network (ALN) model. Each class in the program is delivered on campus via the traditional face to face lecture mode. However, the classes make explicit use of computer and communication technology to facilitate an asynchronous mode of learning for both on-campus as well as distance education students. The lecture and in class interactions including computer demonstrations are captured as video or computer animations. This digital video is compressed and broadcast 'Iive' via the Internet so that students who cannot attend the class can join the lecture. After the class, a student assistant is engaged to generate high quality image and graphical copy of material developed spontaneously in the class by the Instructor. These are then integrated with class notes into a synchronized multimedia presentation.
\end{abstract}

\subsection{INTRODUCTION}

Traditional campus-based engineering education involves a combination of several basic components which serve as sources of knowledge and experience. These include learning media such as textbooks or laboratory manuals, interactions with experts in the field (the instructor or teaching assistant), interactions with fellow learners (student-student interchange), as well as, in some cases, engineering experience such as in laboratory or real world projects. The model of learning which features synchronous interactions between learners, information and experiential sources as well as subject matter experts - namely the traditional campus-based face-to-face education - has served well and endured the test of a great deal of time.

More recently, there have been many attempts to offer engineering and other educational experiences at a distance using written or printed correspondence via regular or courier mail, video and audio tapes, and even one-way and/or two-way video and audio transmissions. While these 'distance learning' strategies have served to provide effective delivery of educational materials and some interaction, the delays incurred and slowness in communication have severely inhibited the success of these methods in capturing the essence of effective learning.

The development and maturing of computer and communication technology have now made it possible to provide all the basic components of the tradition classroom-based learning experience using local computer networks for on- and near-campus access, or the Internet for global access, to these educational resources. Now, instead of sending the printed, audio or video media, we transmit the information -appropriately digitized and compressed and provide immediate interactive and collaborative channels and tools for student-student and studentinstructor dialogue.

In this paper we describe the implementation of the "lectures on demand" method using multimedia streaming technologies with the now widely accepted Asynchronous Learning Network (ALN) model.

The remainder of this paper is organized as follows: We provide in the next section a discussion of Asynchrnonous Learning Networks (ALNs) and then we show in Section 3 how the Lectures on Demand approach complements ALNs by introducing the class-room experience in the form of synchronized streaming audio and video. The paper concludes in Section 4 with some comments on the prospects for further developments in this area.

\subsection{ASYNCHRONOUS LEARNING NETWORKS}

As suggested by the term Asynchronous, ALNs cater largely to learners who are not in the same place or time as each other, or with the instructor, but effective Learning is facilitated by the ALN. While it is tempting to imagine that the Network in ALN has reference to the underlying network communication and interconnected computers, the important network in ALNs is rather the network or community of learners, who interact at their time and from their location. Of course the basic idea of learning asynchronously has been around for quite some time. Clearly, learning is asynchronous when one learns from books, audiotapes and videotapes. However, the idea of coupling independent learners separated by distance and time constraints has been much more feasible in the past 
decade, with the emergence of Internet-based communication technologies. Now instead of sending the physical media (such as printed material, magnetic or optical media) through regular mail or courier services, it is now merely necessary to send the digital content, appropriately encoded, to the user. In addition, the learner can now have access to a network of fellow students via Email, mailing lists, bulletin boards and electronic conferencing systems. The World Wide Web has truly revolutionized student access from any place and at any time, to a wealth of multimedia information sources, which can greatly enhance the learning process.

The combination of convenient and appealing content delivery with seamless and easy to use interactive mechanisms for collaboration and mentoring using online communication tools, enable ALNs to imitate some important aspects of ideal learning environments - such as found, for example, in the early learning experiences of infants and kindergartners. We find that collaborative, active learning and discovery of new information - teaching yourself, and teaching as well as learning from others - are key characteristics of this very successful teaching/learning environment. It must however be admitted that both instructors and students alike have become very comfortable with the non-interactive one way transmission information transfer - with limited feedback, mostly in periodic written examinations. In these settings, the instructor appears to be a 'sage on the stage' expounding gospel truths to a passive audience. On the other hand, in an active learning environment, the teacher is more of a 'guide by the side' - encouraging and exciting the students with the thrill of discovery. Our intent is not to promote the replacement of conventional face-to-face teaching and learning with ALNs. Rather, we argue that that ALNs, when well designed, can in fact replicate many of the desirable features of effective face-to-face learning in promoting and facilitating interaction between students and with the instructor by more convenient and often more acceptable asynchronous means.

We firmly believe that the first choice for learning now and in the foreseeable future will be the traditional method: attending a great institution of learning, with learned professors and dedicated graduate and undergraduate students studying and conducting research under the watchful eyes of these academic sages. Nonetheless, it is clear that ALNs can be used as an appropriate complement for such traditional on-campus classes. On the other hand, for that significant segment of the population who cannot attend face-to-face lectures and oncampus programs, ALNs provide a vastly superior learning experience than study-it-yourself distance education. For example, ALNs provide for immediate feedback, on online quizzes, online shared calendar of events, WWW home pages for each student, customized search and study tools, access to grades and progress reports and online assignment submissions. In addition, online WWW resources, course syllabi, class materials, assignments and solutions as well as access to an online community of peers and instructors make for a very desirable learning environment. Indeed, such features in ALNs as bulletin boards and mailing lists which archive the discussion threads, or more sophisticated electronic conferencing systems and chat systems, serve to build an interactive community of learners which looks very much like the active and collaborative learning scenario we painted earlier.

At the University of Florida we initially experimented with a home grown ALN interface to provide conferencing and discussion lists. We used a majordomo mailing list server to which each class member would subscribe at the beginning of the class (with no further instructor intervention), as well as a hypermail interface which archives the mailing list and sorts the discussion by subject and date. We also used a PERL script-based tool, webchat, for online 'live' discussions. We also decided that we should use a common interface for all our online classes to give a consistent look and feel.

Online students would use the standard ALN interface appearing on the main class page and from here they would visit the key areas of the online course. The bulletin board is used to post announcements such as information about the course, the ALN method and the course outline etc. A key element on the bulletin board will also be a class schedule showing what is expected to be covered as the semester progresses, the associated lectures and topical content as well as the assigned reading materials and exercises. The students can then use this as a guide to gauge their progress. The lounge provides various facilities, such as E-mail , mailing lists and electronic chat rooms for interaction between students as well as with the instructor and the teaching assistants (TAs). The Lab is a forum which allows the students to display their own work on a personal web page and to present interim reports as well as final project reports from which the entire class can benefit. It is in the classroom that the online lecture takes place and homework assignments, solutions and class notes are given. The interested reader is referred to [1] for a demonstration of these elements online.

As the development and delivery of online courses progressed, we decided to use a commercial course management tool as an umbrella for hosting our online courses. WebCT [2] was selected although we are now considering several other options. The individual courses are password protected via WebCT and the standard course template is now integrated with WebCT tools by links to such built in functions as bulletin boards and java-based chat clients. Students can also create their own web pages in WebCT and can check on their grades online as well as take some tests online.

In the next section we describe various considerations in the integration under the ALN umbrella of audio and video from traditional lecture style classes, now repackaged for online course delivery. 
3.0 Lectures on Demand - Streaming Video and Audio of In-class Lecture

Many of our graduate engineering courses at the University of Florida are already being delivered to off-campus corporate sites via videotapes made in specially instrumented classrooms. We therefore elected to use this video class room experience to generate our lectures on demand component for integration within the ALN environment.

Initial access to these professionally equipped recording studio-classrooms was quite an advantage and contributed greatly to the initial success of our online courses. In addition, there are many benefits from using the in-class session as the source for the material for the ALN course to be offered online as a supplement to the on campus classes or to distance education students. One key advantage of this approach is that the instructor can teach in any manner with which he or she is comfortable. The technology does not get in the way of effective and tried and tested methods of teaching and learning and we do not ask the instructors to make any major changes in their teaching style. Of course, the Instructor needs to be well organized in his presentation of material, even if the style of teaching uses the "chalk and talk' methodology. There should be all the essential elements of effective teaching such as the use of motivational and practical examples, and possibly class exercises to get the students involved. Furthermore, we also gain efficiency from the fact that the ALN lectures are being generated during the actual class delivery and does not require several hours of very valuable and expensive instructor time. Rather, the majority of the supporting activities to transform a lecture module into a synchronized multimedia presentation in an ALN context, can be accomplished using student assistants and technologists.

We favor the in-class recordings, rather than staging a video production without students, because of their natural fit with our motivation to develop ALN materials at lower cost and in a scalable manner and to be able to keep the courses fresh over time. Furthermore, in this mode we are able do live webcasts of the class so that some students at a distance can join live classes if this is convenient. In-class questions can be useful in the sense that online students may themselves be asking the same questions as their oncampus counterparts, and thus can benefit from the spontaneous and unchoreographed explanation of the instructor. Of course, online students can also be encouraged to submit spontaneous questions to the class bulletin board or mailing list as these arise in their mind as they watch the lectures on demand courses.

\subsection{Video and Audio Capture - Digitization and Compression \\ In this project[3], we initially elected to use the} RealNetworks version 5.1 encoder, which accepts NTSC video via a Video Capture Card and audio via an industry standard sound card and generates a digital video stream at about $20 \mathrm{kbps}$. A 50-minute lecture using the RealNetworks
5.1 format at $20 \mathrm{kbps}$ required about $9 \mathrm{Mb}$ of data storage compared to $1.2 \mathrm{~GB}$ for an AVI file for the same lecture. The $20 \mathrm{kbps}$ stream was selected because this could readily be received by a user connected to the Internet via a dial-up link at 28.8 or $33.6 \mathrm{kbps}$.

More recent versions of the RealNetworks encoding system - the G2 system - offer the possibility of combining multiple encoding speeds into a single file using the SureStream system. In this mode, the resulting encoded video file is somewhat larger, but now a user who has greater bandwidth available will obtain the higher data rate stream and the data rate will adapt to the connection conditions as necessary. Other recent products such as Microsoft Media and Quicktime 4.0 provide alternatives to the RealNetworks solution; however, the RealNetworks product is currently better known and recognized in this area of application.

Some would argue that the video information is not useful for online delivery of lectures and so may be discarded entirely. We argue that in engineering courses, the fact that spontaneously written material is also transmitted in the video window justifies the inclusion of the video facility. The dynamic effect of drawing a figure or deriving an equation has pedagogical and psychological value and has learning benefits which are lost when a pre-drawn figure or an already written equation is presented on a PowerPoint slide.

Apart from the novelty factor, a digital video window on a computer screen on its own would be unlikely to fare better in terms of educational value and impact than the use of television. The first step in generating a truly effective learning environment is to realize that the video content is supplemental to many other learning facilities in the ALN offering. There are course readings, WWW sites with lecture notes or other relevant material as well as online interactive forums for electronic conferencing. So it is desirable to integrate the streaming video and audio with these other ALN material. When taken together, the streaming video and audio - the lecture on demand - can be the focus of the ALN activities or it could be just one of several components to aid in asynchronous learning.

\subsection{Synchronizing with Spontaneously Generated in- class Notes}

In order to provide an enhancement to the simple streaming video and audio, the lectures on demand methodology includes high quality PowerPoint images generated from the course material presented in class by the instructor. Since the instructor is allowed to teach in any mode with which he is comfortable, the raw course materials may be available in a wide variety of formats, including notes on a white or chalk board or word processor documents, etc. These notes are first formatted into a standard PowerPoint interface and then are saved as HTML documents and images. The video and audio streams are then edited using appropriate software provided by RealNetworks to insert 


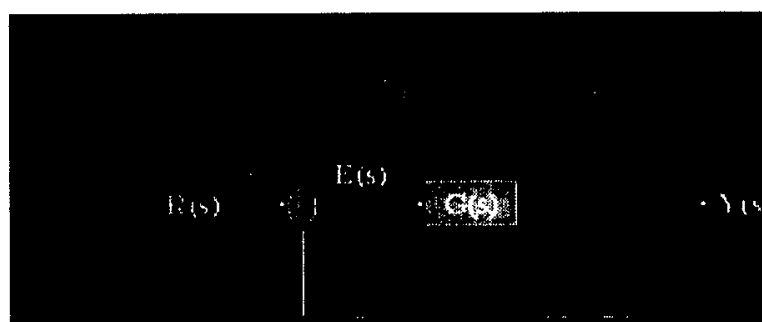

What is the steady state error? $E(t)=r(t)-y(t)$
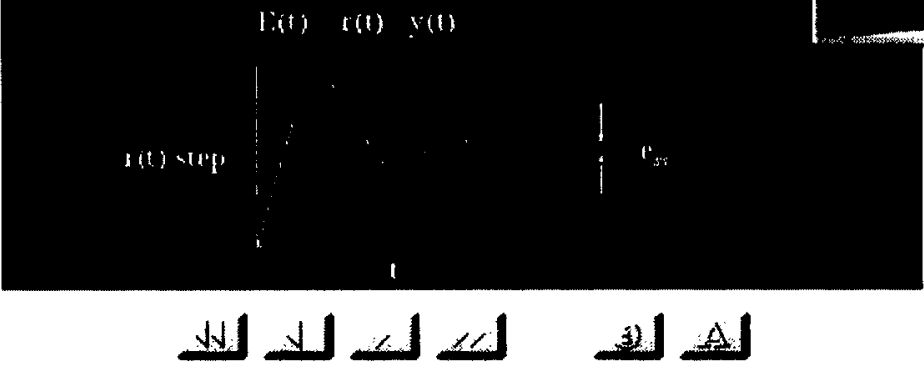

Slide 3 of 7 
Note that in Figure 1, the video window is an inset in the browser window showing the lecture notes. The student who is taking the class online would simply go to the class WWW site and select the lecture desired. As the lecture begins, a new WWW browser window is opened for displaying class notes synchronized with the streaming video. The RealPlayer can be set to be 'always on top while playing' or it can be placed in the background or even minimized if desired. As the lecture proceeds, the lecture notes window advances to the correct lecture notes being discussed in the class. Of course the student is also able to print the lecture notes and to make his or her own notes as the lecture proceeds.

Note that while the slides advance automatically as the lecture progresses, the student still maintains control. Not only can he or she pause, advance, rewind, stop or restart the streaming presentation, but it is also possible to navigate among the slides by using the forward and reverse buttons. In addition, having the streaming video as an independent window allows the student to move, resize or even minimize the video window at will. In particular, if the video content at a certain point of the lecture is not particularly beneficial, the student can place the video window in the background and concentrate on other learning components - such as voice explanations or the PowerPoint slides.

An alternative method of integrating the video window with other www-based class material is to use the concept of frames as illustrated in Figure 2. In this case, the video window is fixed in a particular frame - usually the upper left corner of the screen. The user still has access to certain pre-defined controls but cannot now minimize that particular window. The streaming video and audio can still be synchronized to other www-based materials in other frames. Figure 2 also shows another powerful feature of the RealNetworks system, namely the facility for non-linear navigation of online lecture topics using a modular index of topics which are then linked to particular points in the lecture. In our example of Figure 2, if the student does not need to listen to the entire lecture, but merely needs to get a better understanding of a particular concept, the student would simply click on that link in the index. The effect is to advance the video to that point in the appropriate section of the lecture, which in turn displays the correct auxiliary material in the adjacent window.

\subsection{CONCLUSION}

The lectures on demand in ALN method has proven to be quite effective in extending the classroom experience to a "learning anytime anywhere" educational context. We anticipate that further developments in the area of effective online learning will involve extending the collaborative and active learning experiences of face-to-face classes using group and individual exercises on the WWW using Java applets or other simulation tools. We have already begun to develop some online simulations and virtual experiments.
Our preliminary results so far are encouraging and this will be the subject of a future paper.

\section{ACKNOWLEDGEMENTS}

The work described in this paper was supported in part by a grant from the Alfred Sloan Foundation as well as from the Southeastern University and College Coalition for Engineering Education (SUCCEED), funded by the National Science Foundation.

\section{REFERENCES}

[1] Online ALN Demonstration at http://csc.list.ufl.edu/5718/courseware/index $1 . h t m l$

[2] WebCT Company http://www.webct.com

[3] Latchman, Haniph A., C. Salzmann, D. Gillet, H. Bouzekri, "Information Technology Enhanced Learning in Distance and Conventional Education", IEEE Transaction on Education, November, 1999. 